\title{
Controlled Joint Inflammation but Still No Remission? It's Time to Attend to Depressive Symptoms
}
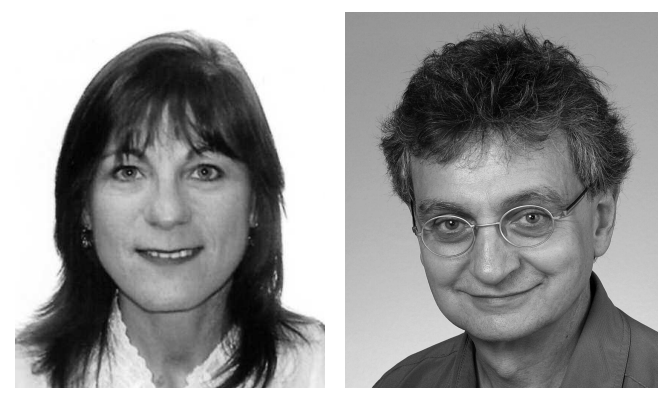

Current guidelines recommend treating patients with rheumatoid arthritis (RA) by targeting remission, or if that fails, low disease activity, providing explicit directives for pharmacological interventions when arthritis remains active ${ }^{1}$. Similar recommendations are being proposed for psoriatic arthritis $^{2}$. Definitions of disease activity in immune-mediated inflammatory arthritides (e.g., Simple Disease Activity Index in RA) incorporate objective [e.g., C-reactive protein (CRP) levels] and physician-derived measures of inflammation [e.g., swollen joint counts (SJC), physician's global assessment of disease activity (PGA)] along with patient-derived ones [e.g., tender joint counts, patient's global assessment of disease activity (PtGA) $]^{3}$. Because of ever-increasing numbers and better use of effective biologic and nonbiologic antirheumatic drugs (DMARD), remission should represent a reasonable goal for every patient with chronic inflammatory arthritis.

Unfortunately, control of inflammation does not directly translate into remission. Rheumatologists confidently use their PGA (and SJC) as their gold standard for treatment decisions regarding DMARD ${ }^{4}$, but remain puzzled when the inflammation is well controlled, but the patient still feels ill ${ }^{5}$. Persistent pain, fatigue, functional limitations, and psychological factors all contribute to elevated PtGA values in patients with controlled inflammation ${ }^{6}$. Ferreira, et al recently described this disconnect as 2 complementary domains needing distinct approaches: (1) control of inflammation, reflected by CRP and SJC, using DMARD; and (2) effect of disease indicated by elevated PtGA values, using adjunctive therapies yet to be defined ${ }^{6}$.

There are biological, behavioral, and psychological reasons for the connection between inflammatory arthritis and affective disorders. Elevated levels of proinflammatory cytokines (e.g., interleukin 6 and tumor necrosis factor- $\alpha$ ) limit psychological adaptation to disease, and when persistent, induce central pain sensitization ${ }^{7}$. Patients with inflammatory arthritis usually have chronic pain and impaired function for several months to years before we diagnose and treat them. Depression in patients with controlled disease may thus have been preexistent, amplified, or induced by their inflammatory condition. Together with socioeconomic status, physical activity, comorbidities such as obesity, and education levels, affective factors are known to influence perception of pain, functional disability, and health status, with strong effect on the use of health resources and on adherence to treatments ${ }^{8}$.

We reported the longterm detrimental role of persistent depression in a cohort of patients with early inflammatory polyarthritis (EPA) who were treated with the aim of zero swollen joints ${ }^{9}$. In that cohort, disease activity rapidly decreased and remained very low thereafter. Similarly, using the RA-adjusted threshold $(\geq 19)$ of the Center for Epidemiologic Studies Depression Scale (CES-D) scores, the rate of depression rapidly decreased from $45 \%$ at baseline to $24 \%$ at 1 year, but then remained at around $19 \%$ over 5 years. These numbers mirror the estimated $16.8 \%$ (95\% CI 10-24\%) prevalence for major depressive disorder in established RA ${ }^{10}$. Of note, over $80 \%$ of those remaining with elevated CES-D scores over 5 years had concomitant SJC of 2 or less. In these patients with EPA, CES-D scores at 12 months into disease accounted for about one-fourth of the variance for nonremission by 5 years, outperforming concomitant SJC, RA antibodies, and even functional limitations ${ }^{9}$. Because patients with established inflammatory arthritis are known to be less responsive to treatment than patients with EPA, higher rates of remission are thus unlikely in patients with treated-to-target inflammatory arthritis if we continue to ignore their persistent depressive symptoms once inflammation is brought under control.

\section{When to Treat Depression in Inflammatory Arthritides} Diagnoses of active arthritides and of depression rely, in part, on subjective assessments that may be affected by biological manifestations of the diseases. Fatigue, sleep perturbations, and poor appetite are shared features of both depression and active RA. Although psychological interventions might be helpful at any stage, controlling inflammation is highly effective at reducing depression in patients with arthritis ${ }^{9,11,12}$. Thus, to be able to evaluate the independent contribution of psychological interventions on patient outcomes, the inflammatory disease must first be treated thoroughly until inflammation is controlled and DMARD treatment is stable.

\section{Screening and Treatment Challenges}

Depressed patients may not disclose their feelings to their rheumatologist, who is seen as a "joint" specialist. In a survey of 226 rheumatologists, only $9 \%$ used a screening

Personal non-commercial use only. The Journal of Rheumatology Copyright @ 2018 . All rights reserved. 
tool $^{13}$, despite the availability of several tools that are quick and easy to administer and score (e.g., CES-D), with or without a nurse assistant. Most rheumatologists did not feel confident about managing mental health issues on their own, nor did they feel it was their role to do so. Rheumatologists indicated that patient resistance to diagnosis and treatment of mental health issues (65.8\%) was the most significant barrier for treatment of depression in their patients, followed by the lack of insurance coverage (46.7\%) and the unavailability of resources $(27.1 \%)$.

Antidepressive medications are the most frequently used approach in the general population, and the most effective in severely depressed patients ${ }^{14,15}$. Yet there are drawbacks to antidepressive medications in chronic inflammatory joint diseases. First, patients are often reluctant to take them, cannot tolerate their side effects (fatigue, weight gain), or refuse to add more medications to the mix they already take to treat arthritis and comorbidities. Second, physician's expertise is necessary because the types and doses required vary across patients. Finally, there is no empirical evidence showing that antidepressants are effective to treat the mild to moderate depression most often found in controlled patients.

Nonpharmacologic interventions have few if any side effects and thus may be more attractive to patients ${ }^{16}$. Exercise may improve depression in arthritic patients, although the evidence is stronger for fibromyalgia ${ }^{17}$. Cognitive-behavioral therapy (CBT) is effective for chronic pain associated with various chronic diseases, including $\mathrm{RA}^{18}$.

Emerging evidence indicates that mindfulness-based interventions may be preferable for the full range of symptoms found in patients with inflammatory arthritis (morning stiffness, fatigue, pain, depression, anxiety). Mindfulness-based stress reduction (MBSR), a standardized 8-week program, has been shown by Zautra, et $a l^{19}$ to be more beneficial than CBT in RA patients with a history of depression. Improvement of patient outcomes, especially the patient-reported ones, is possible with MBSR despite persistently active disease ${ }^{20}$. The program consists of meditation, yoga exercises, coping with illness, and stress management components, offered in a group setting. Thus, MBSR appears to be a promising therapeutic approach in our patients living in a health system with limited psychological resources available.

\section{What Rheumatologists Need to Do}

First, we need to increase rheumatologists' awareness of the importance of treating depression in patients with inflammatory arthritis, especially when depressive symptoms persist once joint inflammation is controlled. Rheumatologists are in the ideal position to identify those patients in need of intervention. Rapid and reliable screening tools for depression are available. Second, once depressive symptoms are identified, rheumatologists and their nurse assistants should help set up collaborative interventions with primary care physicians and other healthcare practitioners, such as psychiatrists, physical therapists, and psychotherapists. Educating patients on how depression, stress, pain, and immune functioning are related and how dealing with emotions may improve quality of life can mitigate the stigma attached to mental health issues and concomitant treatments. In the absence of strong evidence for any one intervention, treatment should be tailored to the patient's personal situation (medications being more effective in severely affected ones ${ }^{15}$ ) and preferences. Despite evidence suggesting superiority for MBSR over CBT, one needs to keep in mind that not all patients are interested in participating in mindfulness-based interventions. For some, the group may be a draw to help alleviate isolation, but it may not appeal to others. Finally, cost may be an issue - group interventions are more affordable than individual psychotherapies.

Along with the use of optimal treat-to-remission strategies designed to attain and maintain control of joint inflammation, rheumatologists need to identify depressive symptoms in their "controlled" patients. More research is required to define which interventions targeting depressive symptoms are most effective in patients with controlled inflammatory arthritis. Nonetheless, this uncertainty should not prevent rheumatologists from taking action now. Existing low-cost, low-risk (e.g., relative to biologic DMARD) psychological interventions have the potential to lead to increased rates of sustained remission and improved longterm quality of life in our patients with chronic arthritis.

\footnotetext{
PATRICIA L. DOBKIN, $\mathrm{PhD}$

Department of Medicine, and

Faculty of Medicine,

Programs in Whole Person Care, McGill University,

Montreal;

GILLES BOIRE, MD, MSc,

Department of Medicine,

Faculty of Medicine and Health Sciences, Université de Sherbrooke, and Division of Rheumatology, Centre intégré universitaire de Santé et de Services Sociaux de l'Estrie, Centre Hospitalier Universitaire de Sherbrooke, Sherbrooke, Quebec, Canada.
}

Address correspondence to Dr. G. Boire, Department of Medicine, CIUSSS de l'Estrie - CHUS, 3001 12th Ave. North, Division of Rheumatology, Room 3853, Sherbrooke, Quebec J1H 5N4, Canada. E-mail: gilles.boire@usherbrooke.ca

\section{REFERENCES}

1. Smolen JS, Landewé R, Breedveld FC, Buch M, Burmester G, Dougados $\mathrm{M}$, et al. EULAR recommendations for the management of rheumatoid arthritis with synthetic and biological disease-modifying antirheumatic drugs: 2013 update. Ann Rheum Dis 2014;73:492-509.

2. Coates LC, Moverley AR, McParland L, Brown S, Navarro-Coy N,

Personal non-commercial use only. The Journal of Rheumatology Copyright $\subset$ $\subset$ 2018. All rights reserved 
O'Dwyer JL, et al. Effect of tight control of inflammation in early psoriatic arthritis (TICOPA): a UK multicentre, open-label, randomised controlled trial. Lancet 2015;386:2489-98

3. Aletaha D, Landewé R, Karonitsch T, Bathon J, Boers M, Bombardier C, et al. Reporting disease activity in clinical trials of patients with rheumatoid arthritis: EULAR/ACR Collaborative Recommendations. Arthritis Rheum 2008;59:1371-7.

4. Pyne L, Bykerk VP, Boire G, Haraoui B, Hitchon C, Thorne JC, et $\mathrm{al}$; CATCH Investigators. Increasing treatment in early rheumatoid arthritis is not determined by the disease activity score but by physician global assessment: results from the CATCH study. J Rheumatol 2012;39:2081-7.

5. Vermeer M, Kuper HH, van der Bijl AE, Baan H, Posthumus MD, Brus HL, et al. The provisional ACR/EULAR definition of remission in RA: a comment on the patient global assessment criterion. Rheumatology 2012;51:1076-80

6. Ferreira RJO, Duarte C, Ndosi M, de Wit M, Gossec L, da Silva JAP. Suppressing inflammation in rheumatoid arthritis: Does patient global assessment blur the target? A practice-based call for a paradigm change. Arthritis Care Res 2017 May 23 (E-pub ahead of print).

7. Altawil R, Saevarsdottir S, Wedrén S, Alfredsson L, Klareskog L, Lampa J. Remaining pain in early rheumatoid arthritis patients treated with methotrexate. Arthritis Care Res 2016;68:1061-8.

8. Hawker GA. The assessment of musculoskeletal pain. Clinic Exp Rheumatol 2017;35 Suppl 107:8-12.

9. Leblanc-Trudeau C, Dobkin PL, Carrier N, Cossette P, De Brum-Fernandes A, Liang P, et al. Depressive symptoms predict future simple disease activity index scores and simple disease activity index remission in a prospective cohort of patients with early inflammatory polyarthritis. Rheumatology 2015;54:2205-14.

10. Matcham F, Rayner L, Steer S, Hotopf M. The prevalence of depression in rheumatoid arthritis: a systematic review and meta-analysis. Rheumatology 2013;52:2136-48.

11. Rathbun AM, Reed GW, Harrold LR. The temporal relationship between depression and rheumatoid arthritis disease activity, treatment persistence and response: a systematic review. Rheumatology 2013;52:1785-94.
12. Kekow J, Moots R, Khandker R, Melin J, Freundlich B, Singh A. Improvements in patient-reported outcomes, symptoms of depression and anxiety, and their association with clinical remission among patients with moderate-to-severe active early rheumatoid arthritis. Rheumatology 2011;50:401-9.

13. Heiman E, Kravitz RL, Wise BL. Rheumatologists' approaches to diagnosis and treatment of depression. J Clin Rheumatol 2016;22:307-11.

14. Fournier JC, DeRubeis RJ, Hollon SD, Dimidjian S, Amsterdam JD, Shelton RC, et al. Antidepressant drug effects and depression severity: a patient-level meta-analysis. JAMA 2010;303:47-53.

15. Feist KM, Hitchon CA, Bernstein CN, Peschken CA, Walker JR, Graff LA, et al. Systematic review and meta-analysis of interventions for depression and anxiety in persons with rheumatoid arthritis. Rheumatology 2013;52:1785-94.

16. Houle J, Villaggi B, Beaulieu MD, Lespérance F, Rondeau G, Lambert J. Treatment preferences in patients with first episode depression. J Affect Disord 2013;147:94-100.

17. Kelley GA, Kelley KS, Hootman JM. Effects of exercise on depression in adults with arthritis: a systematic review with meta-analysis of randomized controlled trials. Arthritis Res Ther 2015;17:21.

18. Sharpe L. Psychosocial management of chronic pain in patients with rheumatoid arthritis: challenges and solutions. J Pain Res 2016;9:137-46.

19. Zautra AJ, Davis MC, Reich JW, Nicassario P, Tennen H, Finan P, et al. Comparison of cognitive behavioral and mindfulness meditation interventions on adaptation to rheumatoid arthritis for patients with and without history of recurrent depression. J Consult Clin Psychol 2008;76:408-21.

20. Fogarty FA, Booth RJ, Gamble GD, Dalbeth N, Consedine NS. The effect of mindfulness-based stress reduction on disease activity in people with rheumatoid arthritis: a randomised controlled trial. Ann Rheum Dis 2015;74:472-4.

J Rheumatol 2018;45:585-7; doi:10.3899/jrheum.170801 